ORIGINAL ARTICLE

\title{
Tendon injury and repair after core biopsies in chronic Achilles tendinosis evaluated by serial magnetic resonance imaging
}

\author{
A Shalabi, L Svensson, M Kristoffersen-Wiberg, P Aspelin, T Movin
}

Br J Sports Med 2004;38:606-612. doi: 10.1136/bjsm.2003.007609

See end of article for authors' affiliations .....................

Correspondence to: Dr Shalabi, Karolinska Institute, Department of Radiology, Center for Surgical Sciences, Division of Radiology, Huddinge University Hospital, Stockholm SE-141 86 , Sweden; Adel.shalabi@ cfss.ki.se

Accepted 29 July 2003
Objective: To evaluate the morphological response and healing process after transverse ultrasound guided core biopsies in chronic Achilles tendinosis using serial magnetic resonance imaging (MRI) over a period of one year.

Methods: The study included 10 patients. Six had five transverse core biopsies and were longitudinally evaluated by MRI before the biopsies and then after one week, three months, seven months, and one year. These patients started a three month eccentric training programme one to two weeks after the biopsy. Four "non-biopsied" and untreated patients were used for comparison. The clinical outcome was categorised according to the level of pain and performance.

Results: The MRI one week after the biopsies showed an increase in tendon volume (T1-WI) and mean signal intensity (PD-WI) of $29 \%$ and $30 \%(p=0.04)$. During follow up, tendon volume and mean signal intensity gradually decreased. One year after the biopsy, the tendon volume had decreased by $20 \%$ and the intratendinous signal by $28 \%$ compared with the index MRI $(p=0.04)$. The untreated patients showed an increase in both tendon volume $(39 \%, p=0.06)$ and intratendinous signal $(37 \%, p=0.14)$ at the one year follow up. After one year, pain and performance had improved in the treated patients but not the untreated patients.

Conclusion: Five transverse ultrasound guided core biopsies induced a lesion in the diseased Achilles tendon. Alterations during healing such as tendon size and intratendinous signal intensity could be evaluated by MRI. The tendon alterations had decreased one year after the core biopsies.
A chilles tendon problems are among the most common and severe conditions affecting athletes in sports requiring running and jumping. ${ }^{1}$ Excessive load with repetitive micro-trauma is the major cause. ${ }^{2}$ However, sedentary and elderly persons also present with Achilles tendon problems.

Imaging methods can be helpful in evaluating the extent of tendon disease and in following the healing process. Magnetic resonance imaging (MRI) and/or ultrasound (US) are regarded as the diagnostic methods of choice in patients with chronic Achilles tendinosis. US is inexpensive, but requires a meticulously performed examination. MRI gives excellent soft tissue contrast, high spatial resolution, and multiplanar capability, making it superior to other examination modalities for depiction of tendon anatomy and pathological changes.

There is increasing interest in the use of MRI to monitor and follow up tendon healing. The healing process after surgically repaired Achilles tendon rupture has been followed by MRI studies. ${ }^{4}$ The development of repair scar tissue after harvesting the central third of the patellar tendon has also been studied using serial MRI investigations. ${ }^{5}$ However, the sequential MRI signal alterations over time in tendon injury and repair need further evaluation.

One suggested rationale of the surgical treatment of chronic Achilles tendinosis is that the excision and splitting of the tendon induces a biological healing process in the diseased tendon by stimulating the remaining viable cells to initiate cell matrix response and healing. ${ }^{67}$

We have previously used the diagnostic longitudinal US guided core biopsy approach to obtain tendon material for morphological studies and pathoanatomic diagnosis. ${ }^{8}{ }^{9}$ This technique induces an apparently limited tendon injury with minor symptoms such as stiffness and tenderness, which cease after a median of five days. ${ }^{8}$ We modified the biopsy technique in this study to the transverse approach, to make sure that the specimen included pure tendon tissue, as biopsy specimens obtained by the longitudinal approach may also include peritendinous tissue.

A case report, with MRI evaluation at a 6 month follow up, of an intratendinous Achilles tendon lesion with acute clinical onset reported regress of the lesion after non-surgical treatment. ${ }^{10}$ Other than this single-patient report, we have not found any report of MRI evaluation of the healing process after acute partial Achilles tendon ruptures. The US guided core biopsy technique applied to the Achilles tendon induces a small standardised acute injury and may serve as a model for understanding the MRI findings over time after non-total Achilles tendon ruptures.

The purpose of this study was to evaluate the morphological response after core biopsies in a limited group of patients with chronic Achilles tendinosis by serial MRI, in terms of tendon volume and intratendinous signal intensity, during a period of one year.

\section{PATIENTS AND METHODS}

Ten patients (seven were men) with chronic Achilles tendinosis were included in the study. All suffered pain and experienced local tenderness on palpation of the mid-portion of the Achilles tendon, $2-7 \mathrm{~cm}$ proximal to the tendon insertion.

Six of these patients (four men) with a median age of 47 years (range 37-54) had five transverse US guided core biopsies. ${ }^{11}$ After one to two weeks, when the symptoms

Abbreviations: MRI, magnetic resonance imaging; US, ultrasound 


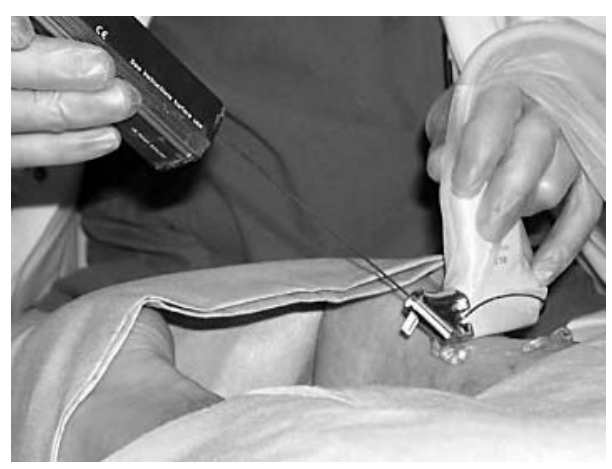

Figure 1 Patient placed in prone position during a percutaneous transverse ultrasound guided core biopsy procedure of the Achilles tendon. The ultrasound transducer in the right hand of the radiologist and core biopsy needle in the left hand.

induced by the biopsy had disappeared, the patients started a three month, heavy loaded, eccentric strengthening programme as described by Alfredson et al. ${ }^{11}$

One male patient had progressive symptoms and had surgical treatment after seven months and was therefore not included in the one year follow up.

The actively treated patients were followed with sequential MRI evaluations: an index examination was performed before biopsy, then one week, three months, seven months, and one year after biopsy.

Four patients (three men; median age of 48 years (range 36-56)) with chronic Achilles tendinosis were examined with MRI. Non-surgical treatment with the eccentric strengthening programme was recommended. However, for different reasons (diabetes mellitus, mild dementia, and, in two instances, illness in the family), these four patients were unable to participate in the eccentric training programme and they did not receive any other specific treatment for their Achilles tendon problems. They were re-examined clinically and by MRI after one year, and thus formed a group of "nonbiopsied" and untreated patients for comparison.

The ethics committee at the Karolinska Institute approved the design of the study, and the patients were informed of the planned procedures before giving their consent.

\section{US guided core biopsy technique}

We modified the biopsy technique to the transverse approach because, besides following the morphological reaction with serial MRI, one reason for the study was to obtain tendinous tissue for quantitative analysis. As longitudinally obtained biopsies may also include peritendinous tissue, a transverse direction was chosen with the aim of obtaining pure tendon tissue. A potential disadvantage of the transverse approach is that several closely performed biopsies may create a partial tendon injury of clinical importance. Therefore no more than five core biopsy samples were taken from each tendon in this study.

The US guided core biopsy examinations were made using an Acuson Sequoia 512 real time scanner with a $13 \mathrm{MHz}$ linear array transducer. The patient was placed in the prone position, and US guided core biopsy samples were taken from the hypoechoic areas in the Achilles tendon (fig 1). Under sterile conditions, a local anaesthetic of the skin, subcutaneous tissue, and fascia, but not the tendon, was applied. An incision of about $2 \mathrm{~mm}$ was made through the skin only.

The US guided biopsy specimens were obtained using a Bard instrument and Bard magnum biopsy needle (CR Bard, Inc, Covington, Georgia, USA) with gradation and an etched tip. The diameter of the needle was $1.2 \mathrm{~mm}$ (14 gauge), the length of the needle $100 \mathrm{~mm}$, and the length of the sample notch $19 \mathrm{~mm}$. The needle was placed inside the Bard instrument and then inserted through the skin and fascia. It was advanced to the surface of the Achilles tendon over the hypoechoic area. The trigger was then fired under US guidance (fig 2).

The location of the hypoechoic area in the Achilles tendon was confirmed by US before removal of the biopsy specimen.

\section{MRI}

The MRI examinations were performed with a Magnetom Vision (Siemens) using a commercially available circular polarized flexible $21 \times 52 \mathrm{~cm}$ coil for all patients. Both Achilles tendons were examined simultaneously.

The images were obtained with the patient in a supine position, the feet kept in a resting position in the coil with a maximum plantar flexion of $15^{\circ}$. The slice thickness was $3 \mathrm{~mm}$ with a $0.3 \mathrm{~mm}$ gap in all sequences. The after sequences were performed in all cases: transversal and sagittal Tl weighted spin echo (TR/TE 730/20 and 550/20, two acquisitions (acq), time of acquisition (TA) 5.54 minutes and 4.45 minutes, field of view (FOV) $200 \mathrm{~mm}$ and $180 \mathrm{~mm}$, and $160 \times 512$ (rec FOV 5/8) and $256 \times 512$ matrix, respectively).

Sagittal FLASH 2D weighted spin echoes (TR/TE 460/10, 2 acq, TA 6.19 minutes, FOV $180 \mathrm{~mm}, 410 \times 512$ matrix) were performed. Sagittal PD/T2 weighted turbo spin echoes (TR/TE $3500 / 17$ and 119,1 acq, TA 4.48 minutes, FOV $180 \mathrm{~mm}$, $400 \times 512$ matrix) were performed. In addition, the first two sequences were repeated after an intravenous contrast agent had been injected.

The contrast agent $(0.2 \mathrm{mmol} / \mathrm{kg}$ body weight gadopentetate dimeglumine; Magnevist; Schering) was given as a bolus within 20 seconds. Immediately after the injection, the catheter was flushed with saline. The two Tl weighted sequences after gadolinium (Gd) contrast agent enhancement (CME) were performed within 10 minutes (CME Tl-WI).
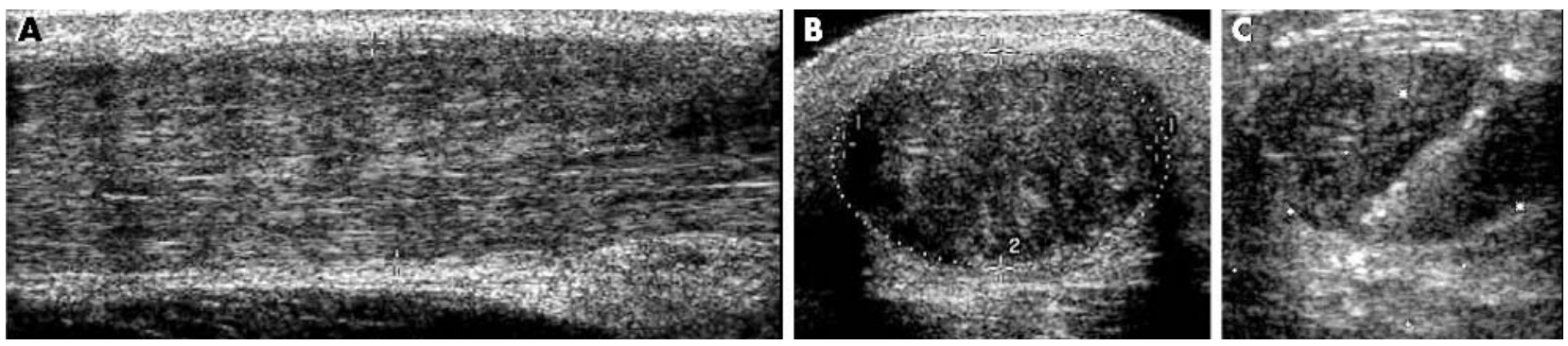

Figure 2 Ultrasonographic longitudinal and transverse images ( $A$ and $B$ ) show the enlarged hypoechoic Achilles tendon. The anteroposterior diameter is $15 \mathrm{~mm}(A)$ and cross section area $2.5 \mathrm{~cm}^{2}$ (B). The transverse image (C) shows the location of the needle inside the tendon tissue after the trigger was fired under ultrasonographic guidance. 

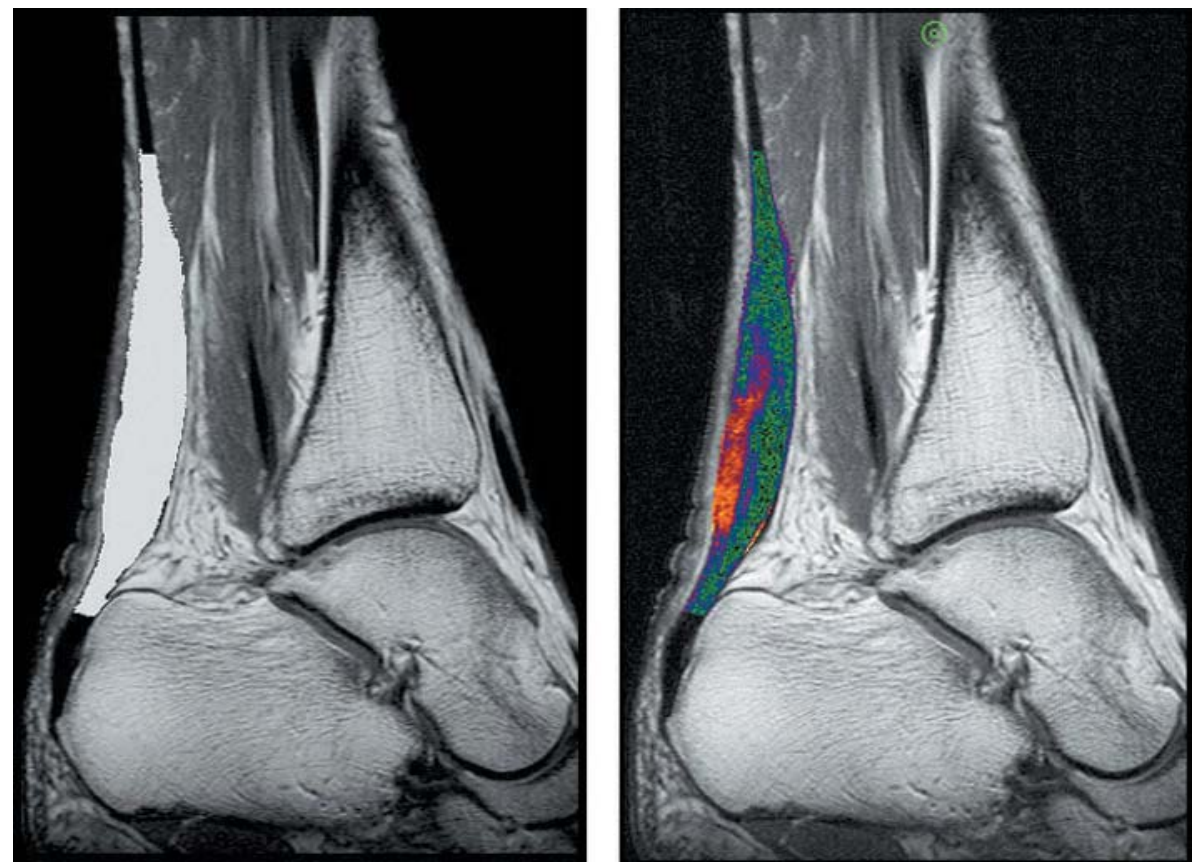

Figure 3 Magnetic resonance images were masked to estimate the volume of Achilles tendon 2-12 cm proximal to the tendon insertion using a seed growing technique. The masked area is superimposed on the standard image in the figure (in white on left and in colour on right).

\section{MRI evaluation}

The volume of the Achilles tendon was evaluated $2-12 \mathrm{~cm}$ proximal to the tendon insertion in the symptomatic and its contralateral tendon and calculated in all sagittal sequences. Seven consecutive sagittal slices formatted as $512 \times 512$ matrices were used to cover each tendon. The pixel size was $0.35 \times 0.35 \mathrm{~mm}^{2}$ and the slice to slice distance $3.3 \mathrm{~mm}$, giving a voxel volume of $0.35 \times 0.35 \times 3.3 \mathrm{~mm}^{3}$ The volume of each tendon was masked using a seed growing technique on a Hermes work station (Nuclear Diagnostics, Sweden). The data set was preprocessed by depicting the $10 \mathrm{~cm}$ interval and masking it to give a $3 \mathrm{D}$ region, including the tendon of interest. A seed was manually marked well within the tendon, and the seed growing algorithm automatically calculated the tendon volume. In some cases, the algorithm leaked out into surrounding tissue. If this occurred, the tendon had to be manually masked. The region grown with this algorithm was used as a binary mask when data for the tendon were extracted from the original data set. The volume of the tendon was calculated by summing all voxels within the tendon and multiplying by the size of the voxel volume.
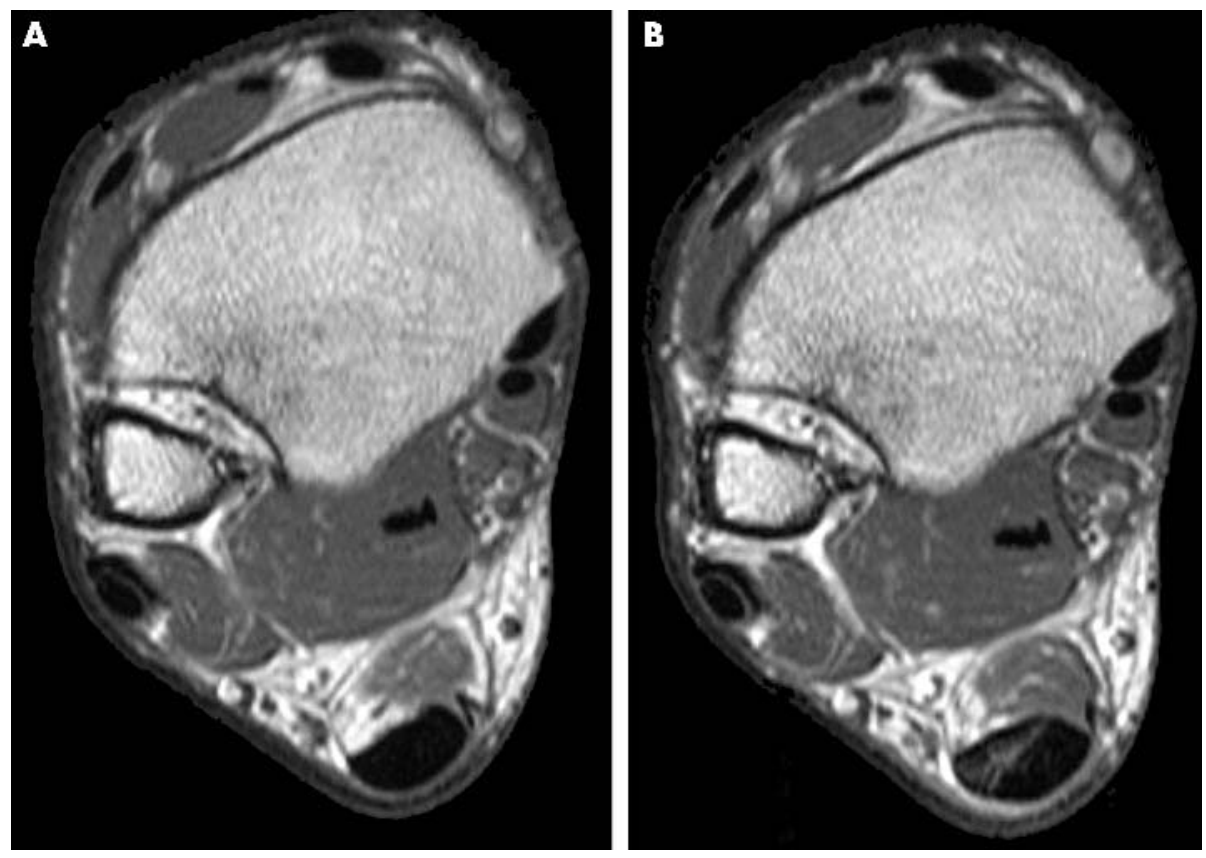

Figure 4 Gadolinium enhanced T1 weighted transverse magnetic resonance images in a 42 year old man showing enlarged Achilles tendon before the biopsy (A). (B) One week after the biopsy, high signal intensity and contrast agent enhancement at the location of the needle biopsy are observed. 

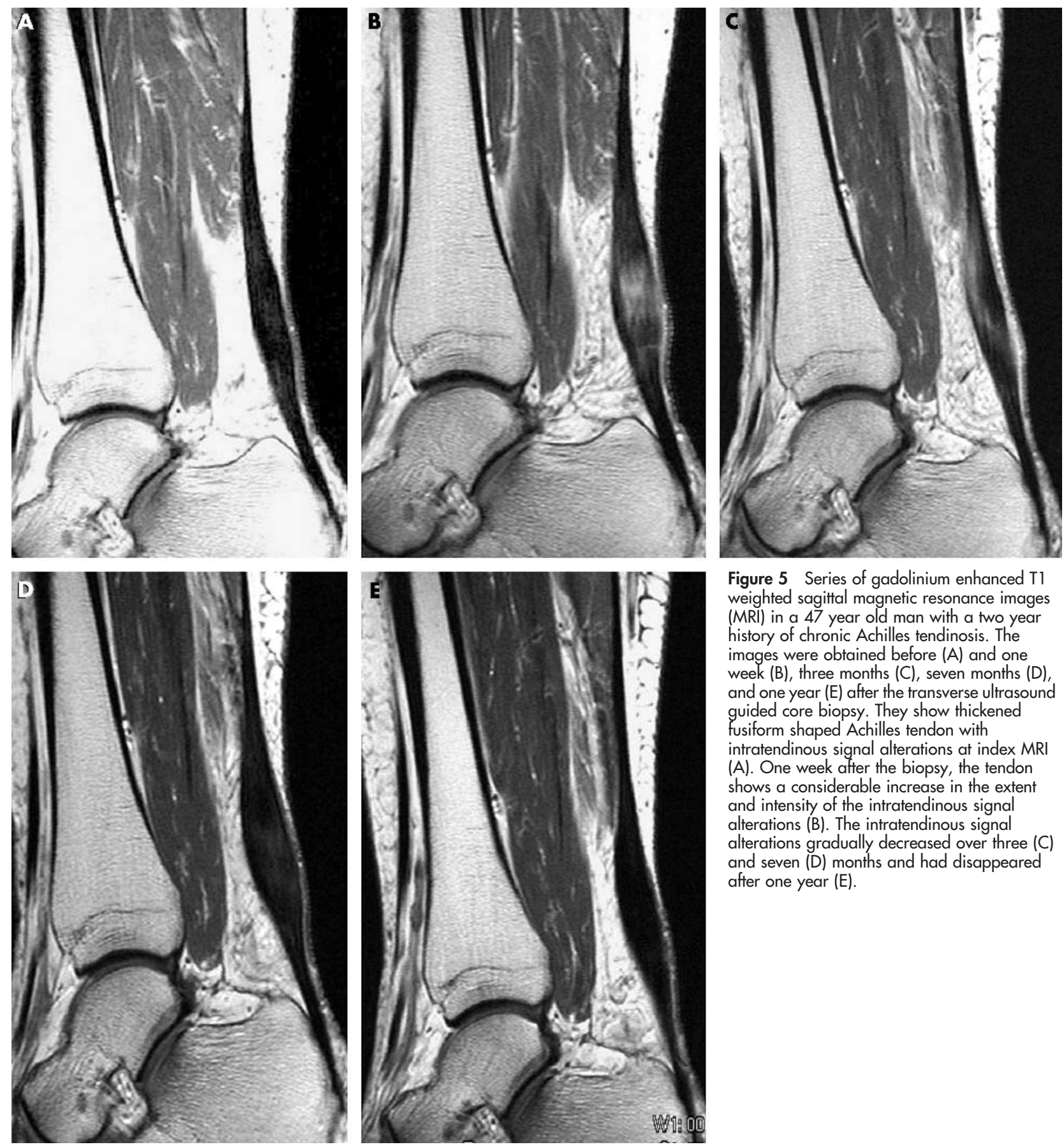

Figure 5 Series of gadolinium enhanced T1 weighted sagittal magnetic resonance images (MRI) in a 47 year old man with a two year history of chronic Achilles tendinosis. The images were obtained before (A) and one week (B), three months $(C)$, seven months (D), and one year (E) after the transverse ultrasound guided core biopsy. They show thickened fusiform shaped Achilles tendon with intratendinous signal alterations at index MRI (A). One week after the biopsy, the tendon shows a considerable increase in the extent and intensity of the intratendinous signal alterations (B). The intratendinous signal alterations gradually decreased over three $(C)$ and seven (D) months and had disappeared after one year (E).

The mean signal of all tendons was estimated from the masked volume (fig 3).

\section{Clinical outcome}

The levels of pain and functional impairment were evaluated at each MRI examination using a questionnaire completed by the patient. The questionnaire used the modified classification of Curwin and Stanish (1984), in which pain was categorised on a six point scale $(l=$ no pain, $6=$ daily pain), and performance on a four point scale $(1=$ normal function, $4=$ unable to participate in sports). ${ }^{3}$

The clinical outcome was categorised as excellent (no symptoms), good (pain associated with extreme exertion only), fair (patients with symptoms in between poor and good), or poor (pain during daily activities, or inability to participate in sports).

\section{Statistical analysis}

Variables of continuous and ordinal types are presented as median and range (minimum-maximum). The non-parametric Wilcoxon matched pairs test was used to compare the tendon volume and intratendinous signal on the MRI index examination with the follow up serial MRI examinations. This test was further used to analyse the clinical outcome. $\mathrm{p}<0.05$ was considered significant.

\section{RESULTS}

The main finding was an initial increase followed by a gradual decrease in the volume and intratendinous signal of the Achilles tendon after the transverse core biopsies. The procedure resulted in minimal skin lesion and intratendinous signal changes which were well depicted after one week on MRI in all patients (fig 4). The tendon volume and mean 
signal intensity had increased one week after the biopsy $(\mathrm{p}<0.05$; tables 1 and 2$)$. The skin lesion was no longer detectable by MRI after three months. However, the alterations in intratendinous signal were still present at the three month MRI evaluation but had further diminished at the seven month evaluation and disappeared after one year (figs 5-7).

There was a significant decrease in tendon volume and intratendinous signal one year after the transverse core biopsy $(\mathrm{p}<0.05$; tables $\mathrm{l}$ and 2 ).

Whereas the tendon volume and intratendinous signal gradually decreased in the actively treated patients, they had increased in the untreated patients at the one year follow up. The tendon volume measured on Tl-WI had increased by $39 \%$ from $6.1(5.2-8.4)$ to $8.5(7.1-15.4) \mathrm{cm}^{3}(\mathrm{p}=0.06)$, and the intratendinous signal on PD-WI had increased by $37 \%$ from $181(154-302)$ to $247(201-421)$ signal units $(p=$ $0.14)$.

The clinical outcome at one year follow up in the actively treated patients was categorised as good to fair. The pain level had reduced (from a median value of 5 (range 5-6) to 3 (range 2-4)), and the performance had improved (from median value of 3 (range $3-4)$ to 2 (range $2-3))(p=0.02$ and 0.03 respectively). In contrast, the clinical outcome remained unchanged or had worsened in the untreated patients at the one year follow up and was categorised as fair to poor.

\section{DISCUSSION}

To our knowledge, there are no previously published studies using serial MRI evaluation of an iatrogenic tendon injury, inflicted with the aim of inducing biological reaction in chronic Achilles tendinosis. The results of our study show healing in a limited group of patients who underwent transverse US guided core biopsy and a heavy loaded eccentric strengthening programme, evaluated by MRI. In addition, the healing process in this group was compared with an untreated group.

This study supports the view that MRI can be used to evaluate tendon healing. Furthermore, it presents unique data from the MRI evaluation of non-total Achilles tendon ruptures. An acute small Achilles tendon tear results in increased tendon volume and intratendinous signal after one week. Our data show that the intratendinous signal then decreases but is still evident on MRI after three months. One

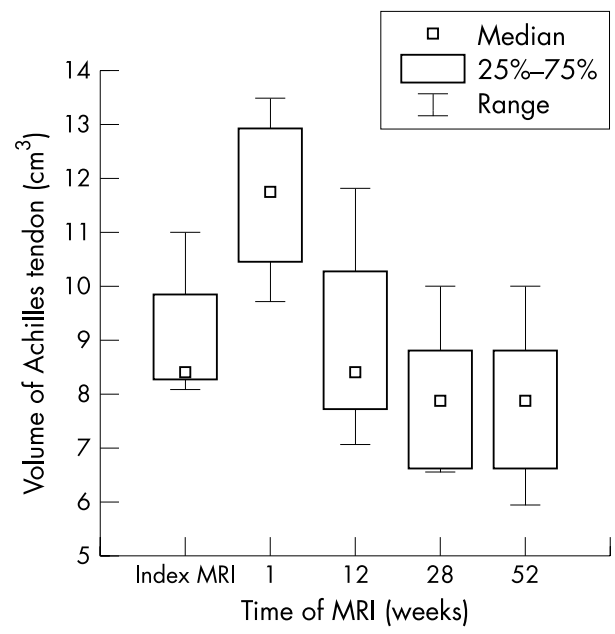

Figure 6 Volume of the Achilles tendon measured on T1-WI images in a magnetic resonance imaging (MRI) series: before and one week, three months, seven months, and one year after ultrasound guided core biopsy. limitation in the comparison with the clinical evaluation is that the tendon injury in our study is small, transverse, and mainly affecting the endotenon. Another limitation is that the healing process is studied using diseased Achilles tendons. Further, the decrease in tendon volume and intratendinous signal may have been influenced by the heavy loaded eccentric strengthening programme.

We have previously used the US guided core biopsy needle technique mainly as a diagnostic and research tool, but theoretically it can be used as a therapeutic method by inducing a biological reaction resulting in repair of the tendon tissue. However, this needs further study and evaluation in a larger prospective series, as our study was limited by the relatively small number of patients.

Eccentric calf muscle training in patients with chronic Achilles tendinosis, as well as minor variations in surgical treatment, have been introduced and used in the last few years. Eccentric strengthening of the gastrocnemius and soleus muscles and loading of the Achilles tendon is important for both prevention and conservative management of chronic Achilles tendinosis. ${ }^{11}{ }^{12}$

Multiple percutaneous longitudinal tenotomies have been used in patients with isolated and well defined nodular lesions (less than $2.5 \mathrm{~cm}$ long) after the precise location of the lesion by US scanning. ${ }^{73}$ More recently, US guided percutaneous longitudinal tenotomy has been used in patients with a single and well defined intratendinous lesion lacking paratenon involvement with a relatively high rate of success. ${ }^{14}$ Further, US guided sclerosis of neovessels in painful Achilles tendinosis has been introduced in a pilot study of new treatment methods. ${ }^{15}$

However, there are some conflicting results on the value of radiological follow up of the healing Achilles tendon in total ruptures. It is unclear whether MRI and US can predict the outcome after treatment of Achilles tendon ruptures. Furthermore, the correlation between clinical parameters and radiological findings appears to be uncertain. Möller et al $^{16}$ reported weak non-significant correlations between the number of positive MRI findings and clinical outcome parameters after one year for patients with Achilles tendon rupture. Maffulli et $a l^{17}$ found that areas of inhomogeneous signals were present in the operated ruptured Achilles tendon in five out of 16 patients after three years. These areas were less than $25 \%$ of the anteroposterior diameter of the tendon and were clinically silent. These findings probably represent

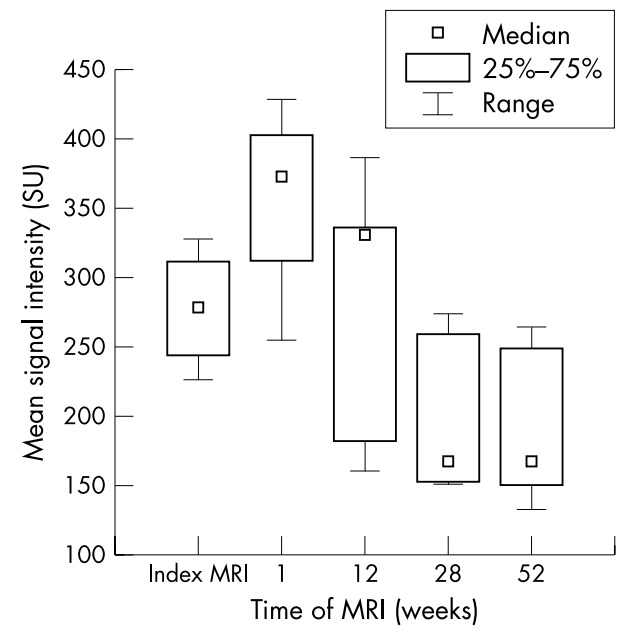

Figure 7 Median signal intensity of the Achilles tendon measured on PD-WI images in a magnetic resonance imaging (MRI) series: before and one week, three months, seven months, and one year after ultrasound guided core biopsy. SU, Signal units. 
Table 1 Volume of the Achilles tendon measured on magnetic resonance images before (MRI 0) and one week (MRI I) and one year (MRI IV) after the transverse core biopsy procedure

\begin{tabular}{lrllllll}
\hline & MRI 0 & MRI I & $\Delta$ volume I & p Value & MRI IV & $\Delta$ volume II & p Value \\
\hline T1-WI & 9.83 & 12.65 & $+29 \%$ & $<0.05$ & 7.87 & $-20 \%$ & $<0.05$ \\
T2-WI & 10.40 & 12.76 & $+23 \%$ & $<0.05$ & 7.96 & $-23 \%$ & $<0.05$ \\
PD-WI & 10.02 & 12.55 & $+25 \%$ & $<0.05$ & 7.69 & $-23 \%$ & $<0.05$ \\
GE-WI & 9.97 & 13.09 & $+31 \%$ & $<0.05$ & 6.95 & $-30 \%$ & $<0.05$ \\
CME T1-WI & 9.53 & 12.43 & $+31 \%$ & $<0.05$ & 7.52 & $-21 \%$ & $<0.05$ \\
\hline
\end{tabular}

The tendon volume in five different sagittal MRI sequences is given in $\mathrm{cm}^{3}$. The percentage change in volume one week ( $\Delta$ volume I) and one year ( $\Delta$ volume II) after the biopsy is also shown. The non-parametric Wilcoxon matched pairs test was used.

Table 2 Magnetic resonance imaging (MRI) intratendinous signal alteration in signal units before (MRI 0) and one week (MRI I) and one year (MRI IV) after the transverse core biopsy procedure

\begin{tabular}{lccccccc}
\hline & MRI 0 & MRI I & $\boldsymbol{\Delta}$ signal I & p Value & MRI IV & $\Delta$ signal II & p Value \\
\hline TI-WI & 104 & 141 & $+36 \%$ & $<0.05$ & 68 & $-34 \%$ & $<0.05$ \\
T2-WI & 101 & 122 & $+21 \%$ & $<0.05$ & 76 & $-25 \%$ & $<0.05$ \\
PD-WI & 270 & 350 & $+30 \%$ & $<0.05$ & 194 & $-28 \%$ & $<0.05$ \\
GE-WI & 227 & 305 & $+34 \%$ & $<0.05$ & 151 & $-33 \%$ & $<0.05$ \\
CME TI-WI & 151 & 217 & $+43 \%$ & $<0.05$ & 95 & $-37 \%$ & $<0.05$ \\
\hline
\end{tabular}

The intratendinous signal alteration in five different sagittal MRI sequences is shown. The percentage change in signal intensity one week ( $\Delta$ signal I) and one year ( $\Delta$ signal II) after the biopsy is also shown. The non-parametric Wilcoxon matched pairs test was used.

normal features of long term tendon healing after open repair of an Achilles tendon rupture. In contrast, Karjalainen et al ${ }^{18}$ noted that two of 13 patients with $1-3$ year old surgically repaired complete rupture of the Achilles tendon, who had the largest intratendinous signal areas, had a poor clinical outcome. Furthermore, Khan et $a l^{19}$ reported that a low grade of MRI signal abnormality before treatment was associated with better clinical outcome after 12 months than a high grade of MRI signal abnormality.

Studies on the healing process of the Achilles tendon are sparse, especially those with a non-rupture cause. ${ }^{4}$ With regard to ruptured Achilles tendons, Karjalainen et $a l^{4}$ described the MRI appearance after surgical repair, with high intensity signal areas at the rejoined tendon ends at six weeks and three months believed to correspond to the healing response. These areas of high intensity signal had diminished or disappeared completely after six months. Tendon thickening is a well known consequence of Achilles tendon rupture. ${ }^{16-18}$

\section{Information box}

- US and MRI are well established in the diagnosis of tendon lesions and other sport injuries. The US guided core biopsy needle technique has been reported as a tool in human tendon research.

- The biopsy procedure in chronic Achilles tendinosis resulted in a limited tendon injury, evident on MRI as increased tendon volume and intratendinous signal after one week. The intratendinous signal then decreased but was still evident on MRI after three months.

- This study supports the hypothesis that MRI can be used to evaluate tendon injury and repair and may be of increasing importance for follow up of treatment interventions in clinical research.
In surgically repaired total rupture, maximal tendon dimensions were found after three months (followed by a decrease). ${ }^{4}$ This agrees with our findings on tendon lesion induced by biopsy and its subsequent repair, resulting in early increase in tendon volume and intertendinous signal intensity followed by gradual decrease.

In conclusion, a technique using five transverse core biopsies induced limited skin and tendon lesions. This procedure initiated a healing process in the diseased tendons. The morphological response and repair in chronic Achilles tendinosis could be evaluated by serial MRI. During the healing process, the increase in tendon volume and extent of intratendinous signal alterations ultimately gradually disappeared. The clinical data indicate that the biopsied and exercised tendons were in a better functional and morphological condition than in the untreated group after one year.

\section{ACKNOWLEDGEMENTS}

This study was supported by a grant from the Swedish National Center for Research in Sports. The help of Dr Laszlo Mihocsa, Department of Radiology, Huddinge University Hospital, Karolinska Institute, is gratefully acknowledged.

\section{Authors' affiliations}

A Shalabi, L Svensson, M Kristoffersen-Wiberg, P Aspelin, T Movin, Karolinska Institute, Stockholm, Sweden

\section{REFERENCES}

1 Kvist M. Achilles tendon injuries in athletes. Ann Chir Gynaecol 199:80:188-201.

2 Åstrom M, Rausing A. Chronic Achilles tendinopathy. A survey of surgical and histopathological findings. Clin Orthop 1995;316:151-64.

3 Rolf C, Movin T. Etiology, histopathology, and outcome of surgery in achillodynia. Foot Ankle Int 1997;18:565-9.

4 Karjalainen PT, Aronen HJ, Pihlajamaki HK, et al. Magnetic resonance imaging during healing of surgically repaired Achilles tendon ruptures. Am J Sports Med 1997;25:164-71.

5 Kartus J, Movin T, Papadogiannakis N, et al. A radiographic and histologic evaluation of the patellar tendon after harvesting its central third. Am J Sports Med 2000;28:218-26.

6 Leadbetter WB. Cell-matrix response in tendon injury. Clin Sports Med 1992; 11:533-78. 
7 Testa V, Maffulli N, Capasso G, et al. Percutaneous longitudinal tenotomy in chronic Achilles tendonitis. Bull Hosp Joint Dis 1996;54:241-4.

8 Movin T, Guntner P, Gad A, et al. Ultrasonography-guided percutaneous core biopsy in Achilles tendon disorder. Scand J Med Sci Sports 1997:7:244-8.

9 Movin T. Tendon tissue sampling. Scand J Med Sci Sports 2000;10:368-71.

10 Nicolaisen T, Skovgaard D, Kjaer M. Conservative treatment of a partial Achilles tendon rupture with an intratendinous lesion. Scand J Med Sci Sports 1997:7:191-3.

11 Alfredson $\mathrm{H}$, Pietila T, Jonsson P, et al. Heavy-load eccentric calf muscle training for the treatment of chronic Achilles tendinosis. Am J Sports Med 1998;26:360-6.

12 Stanish WD, Rubinovich RM, Curwin S. Eccentric exercise in chronic tendinitis Clin Orthop 1986;208:65-8.

13 Maffulli N, Testa V, Capasso G, et al. Results of percutaneous longitudinal tenotomy for Achilles tendinopathy in middle- and long-distance runners. Am J Sports Med 1997;25:835-40.
14 Testa V, Capasso G, Benazzo F, et al. Management of Achilles tendinopathy by ultrasound-guided percutaneous tenotomy. Med Sci Sports Exerc 2002;34:573-80.

15 Öhberg L, Alfredson H. Ultrasound guided sclerosis of neovessels in painful chronic Achilles tendinosis: pilot study of a new treatment. Br J Sports Med 2002;36:173-5.

16 Möller M, Lind K, Movin T, et al. Calf muscle function after Achilles tendon rupture. A prospective, randomised study comparing surgical and nonsurgical treatment. Scand J Med Sci Sports 2002;12:9-16.

17 Maffulli N, Thorpe AP, Smith EW. Magnetic resonance imaging after operative repair of achilles tendon rupture. Scand J Med Sci Sports $2001 ; 11: 156-62$

18 Karjalainen PT, Ahovuo J, Pihlajamaki HK, et al. Postoperative MRI imaging and ultrasonography of surgically repaired Achilles tendon ruptures. Acta Radiol 1996;37:639-46.

19 Khan KM, Forster BB, Robinson J, et al. Are ultrasound and magnetic resonance imaging of value in assessment of Achilles tendon disorders? A two year prospective study. Br J Sports Med 2003;37:149-253. 